\title{
SYNCHROTRON RADIATION AND INSTRUMENTATION AT THE EUROPEAN SYNCHROTRON RADIATION FACILITY
}

\author{
A. KVICK \\ European Synchrotron Radiation Facility, BP 220, 38043 Grenoble Cedex, France
}

\begin{abstract}
A low-emittance $6 \mathrm{GeV}$ storage ring aimed at producing high-brilliance synchrotron radiation from 29 insertion devices and 27 bending magnet ports is being presently constructed in Grenoble, France. The insertion devices include undulators, wigglers and wavelength shifters giving access to high-brilliance undulator radiation as well as extremely high-flux wiggler radiation. The energy of the storage ring will produce fundamental radiation around $1 \AA$ from the undulators whereas the critical energy of the wigglers in some cases approaches $30 \mathrm{keV}$. The wavelength shifters will produce useful radiation well into the hundreds of $\mathrm{keV}$ region. In order to cope with the high thermal load from the insertion devices novel cooling schemes such as cryogenic cooling as well as adaptive mirror technology is being implemented. The European Synchrotron Radiation Facility will start its operation with 7 beamlines in 1994. A total of 30 facility beamlines will be built by 1999 .
\end{abstract}

PACS numbers: $07.85 .+n$

\section{Introduction}

It is well known that charged particles emit electromagnetic radiation when they are accelerated. For particles with non-relativistic velocities the emitted radiation has the $\sin ^{2} \theta$ angular dependence of the Larmor formula. Interesting effects, however, appear when the particles reach relativistic speeds, and over the last few decades storage rings for electrons or positrons have been constructed to exploit this new source of electromagnetic radiation, which is well collimated, intense and extends from the infrared region well down to the hard X-ray region. The basic physics of synchrotron radiation is given by J.D. Jackson [1].

An increasing number of experiments are now performed at the several operating synchrotron radiation facilities, but it is clear that a new dimension in synchrotron radiation research will be added when a number of a new generation dedicated storage rings will come into operation around the middle of the 1990's. This article will briefly outline the basic principles and prospects for synchrotron 
TABLE

ESRF beamlines.

\begin{tabular}{|c|c|c|c|}
\hline & Beam line & Scientific goals & Source \\
\hline 1. & Microfocus & $\begin{array}{l}\text { Microdiffraction } \\
\text { Small-angle scattering } \\
\text { High pressure }\end{array}$ & $\begin{array}{l}\text { Undulator } \\
0.8-3 \AA\end{array}$ \\
\hline 2. & $\begin{array}{l}\text { Multipole wiggler/ } \\
\text { Materials science }\end{array}$ & $\begin{array}{l}\text { Small molecule crystallography } \\
\text { Magnetic scattering } \\
\text { Diffraction }\end{array}$ & $\begin{array}{l}\text { Wiggler } \\
4-60 \mathrm{keV}\end{array}$ \\
\hline 3. & $\begin{array}{l}\text { Multipole wiggler/ } \\
\text { Biology }\end{array}$ & $\begin{array}{l}\text { Laue protein crystallography } \\
\text { Monochromatic option } \\
\text { High pressure ED } \\
\text { Diffraction }\end{array}$ & $\begin{array}{l}\text { Wiggler } \\
4-60 \mathrm{keV}\end{array}$ \\
\hline 4. & $\begin{array}{l}\text { High-flux } \\
\text { beam line }\end{array}$ & $\begin{array}{l}\text { Real-time small-angle scattering; } \\
\text { Monochromatic protein crystallography }\end{array}$ & $\begin{array}{l}\text { Undulator } \\
\text { tunable around } 1 \AA\end{array}$ \\
\hline 5. & $\begin{array}{l}\text { High-energy } \\
\text { X-ray scattering }\end{array}$ & $\begin{array}{l}\text { Gamma-ray diffraction } \\
\text { Small-angle scattering } \\
\text { Compton scattering }\end{array}$ & $\begin{array}{l}\text { Multipole wiggler } \\
\text { or wavelength } \\
\text { shifter }\end{array}$ \\
\hline 6. & $\begin{array}{l}\text { Circular } \\
\text { polarization }\end{array}$ & $\begin{array}{l}\text { Dichroism in EXAFS; SEXAFS; } \\
\text { Spin-dependent photoemission; } \\
\text { Microscopy at } 2.5 \mathrm{keV}\end{array}$ & $\begin{array}{l}\text { Helical undulator } \\
E \leq 4 \mathrm{keV}\end{array}$ \\
\hline 7. & $\begin{array}{l}\text { Surface } \\
\text { diffraction }\end{array}$ & $\begin{array}{l}\text { Surface structural studies; } \\
\text { phase transitions, } \\
\text { growth mechanisms } \\
\text { Liquid surface diffraction }\end{array}$ & $\begin{array}{l}\text { Undulator } \\
K_{\max }=1.85\end{array}$ \\
\hline 8. & $\begin{array}{l}\text { Dispersive } \\
\text { EXAFS }\end{array}$ & Time-resolved structural studies & $\begin{array}{l}\text { Wiggler or } \\
\text { tapered undulator }\end{array}$ \\
\hline 9. & $\begin{array}{l}\text { Undulator } \\
\text { "open" beam line }\end{array}$ & & \\
\hline 10. & $\begin{array}{l}\text { Bending magnet } \\
\text { "open" beam line }\end{array}$ & & \\
\hline
\end{tabular}

radiation research at one of these third generation storage rings under construction: the European Synchrotron Radiation Facility (ESRF) in Grenoble, France.

The new storage rings are characterized by low emittance (small source size and small beam divergence) and the use of so-called insertion devices as radiation sources.

The new storage rings fall into two groups based on the operational energy of the rings. The low-energy rings (1-2 GeV) such as the Advanced Light Source in Berkeley, USA; Elettra, Trieste, Italy; MAX2, Lund, Sweden nad BESSYII in Berlin, Germany are optimized to give radiation in the UV to soft $\mathrm{X}$-ray region. 
The high-energy machines $(6-8 \mathrm{GeV})$ are constructed for the generation of hard $\mathrm{X}$-rays or to optimize the output from the undulators in the vicinity of $1 \AA$. There are three machines in this category under construction: ESRF (6 GeV), Grenoble, APS (7 GeV), Argonne, USA, and Spring-8 (8 GeV) in Japan.

ESRF is a joint project between 12 European countries: France, Germany, United Kingdom, Italy, Spain, Switzerland, Sweden, Norway, Denmark, Finland, Belgium, and Holland. The ring energy of $6 \mathrm{GeV}$ was chosen to provide $14.4 \mathrm{keV}$ photons from the fundamental frequency of an undulator but it also produces high flux and high critical energy $(19 \mathrm{keV})$ from the bending magnets. The storage ring is designed to provide a large number of straight sections to accommodate insertion devices which produce extremely brilliant synchrotron light (photons/s/unit area/unit solid angle/0.1\% bandwidth). This demands a low emittance, which, in the case of ESRF, is 7 nanometer-radians in the horizontal direction and approximately 10 times smaller in the vertical direction. The storage ring will initially operate with electrons as stored particles in up to 992 bunches giving a minimum bunch separation of $3 \mathrm{~ns}$. The bunch length is about $50 \mathrm{ps}$. The beam lifetime should be about 10 hours with initial current of $0.1 \mathrm{~A}$. A positron option can be used if increased beam lifetime is needed. The ESRF will have 29 straight sections, where insertion devices of up to $5 \mathrm{~m}$ in length can be installed. In addition to the straight sections 27 bending magnets may also be used as radiation sources. A detailed account of the ESRF project is given in the ESRF Foundation Phase Report [2]. ESRF will start its operation in 1994 with seven beamlines, which will expand to 20 by 1995 . Funding for a total of 30 facility beamlines have been provided. See Table for a list of the 20 first beamlines.

\section{Insertion devices}

The third generation synchrotron sources are designed primarily to rely on the insertion devices (wigglers and undulators) for the production of the radiation. The principle of an insertion device is to enhance the synchrotron radiation emission as much as possible without disturbing the stored particle beam. The insertion device consists of an array of magnets producing a magnetic field usually in the vertical direction, which is sinusoidal in character in the beam direction. The varying magnetic field introduces horizontal "wiggles" in the electron or positron orbits producing radiation emission along the insertion device. The horizontal excursions are usually a few $\mathrm{mm}$ at most.

The trajectory of the charged particle is horizontal with a transverse velocity $v(x)$ and position $x$ given by

$$
\begin{aligned}
& \frac{v(x)}{c}=\frac{K}{\gamma} \sin \left(2 \pi s / \lambda_{0}\right), \\
& x=\frac{\lambda_{0}}{2 \pi} \frac{K}{\gamma \cos \left(2 \pi s / \lambda_{0}\right)},
\end{aligned}
$$

where $c$ is the speed of light and $\gamma$ is the particle energy divided by its rest energy. $\lambda_{0}$ is the period of the sinusoidal motion and $s$ is the forward direction coordinate. 
The parameter $K$, which is defined as

$K=0.934 \cdot B[\mathrm{~T}] \cdot \lambda_{0}[\mathrm{~cm}]$,

characterises the important features of the insertion devices. $K$ is called the deflection parameter and is the peak angular deviation over $1 / \gamma$ for the insertion device. $1 / \gamma$ is the natural opening angle of the radiation. The division of insertion devices between wigglers and undulators is based on the deflection parameter $K$. Usually a wiggler has a $K$ value of more than 3 , whereas undulators have values less than 3. The wiggler spectrum looks very much alike to bending magnet spectrum, i.e., it has continuously varying intensity distribution over a large energy range. The critical energy

$$
E_{\mathrm{c}}=0.655 \cdot E^{2}[\mathrm{GeV}] \cdot B[\mathrm{~T}]
$$

defines the median energy for the emitted power of the wiggler device. The flux output from a wiggler changes as $2 N$, where $N$ is the number of magnetic periods in the device. It should be noted that the horizontal divergence may be up to a few milliradians $(2 K / \gamma)$. Insertion devices with very high $K$ values are called wavelength shifters and have few periods and very high critical energies.

In the undulator regime the insertion device characteristics are dominated by the interference effects along the $N$ periods. Constructive interference occurs at the resonant wavelengths when the light moves exactly one period ahead of an electron crossing a single magnetic period. In contrast to the wiggler the undulator radiation peaks at these wavelengths (or harmonics) creating a spectrum with marked peaks. Due to the interference the flux of an undulator changes as $N^{2}$ rather than as $2 N$. Typical fluxes from ESRF insertion devices are given in Fig. 1 and 2. A technical review of ESRF insertion devices was given by Elleaume [3].

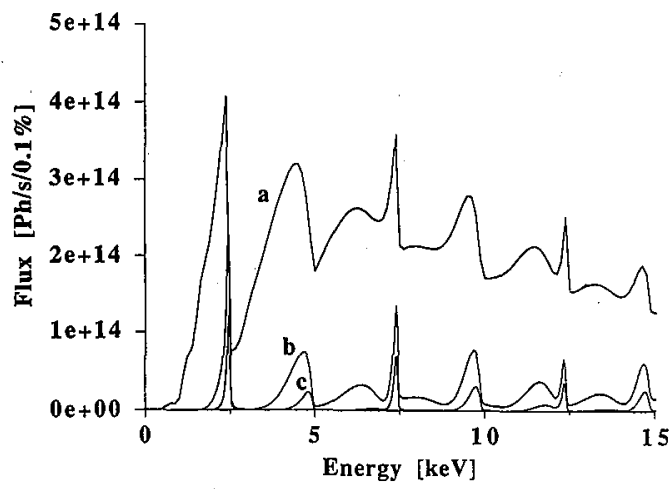

Fig. 1. The radiation spectrum from an ESRF undulator of length $1.6 \mathrm{~m}$ with a period of $46 \mathrm{~mm}$ and $K=2.00$. The figure illustrates the flux at $30 \mathrm{~m}$ from the undulators. The fundamental and harmonics up to order 16 are included. (a) $7 \times 15 \mathrm{~mm}^{2}$ pinhole, power $=650 \mathrm{~W}$, power density $=6.2 \mathrm{~W} / \mathrm{mm}^{2}$; (b) $1 \times 5 \mathrm{~mm}^{2}$ pinhole, power $=78 \mathrm{~W}$, power density $=16 \mathrm{~W} / \mathrm{mm}^{2}$; (c) $0.75 \times 1.5 \mathrm{~mm}^{2}$ pinhole, power density $=22 \mathrm{~W} / \mathrm{mm}^{2}$. 


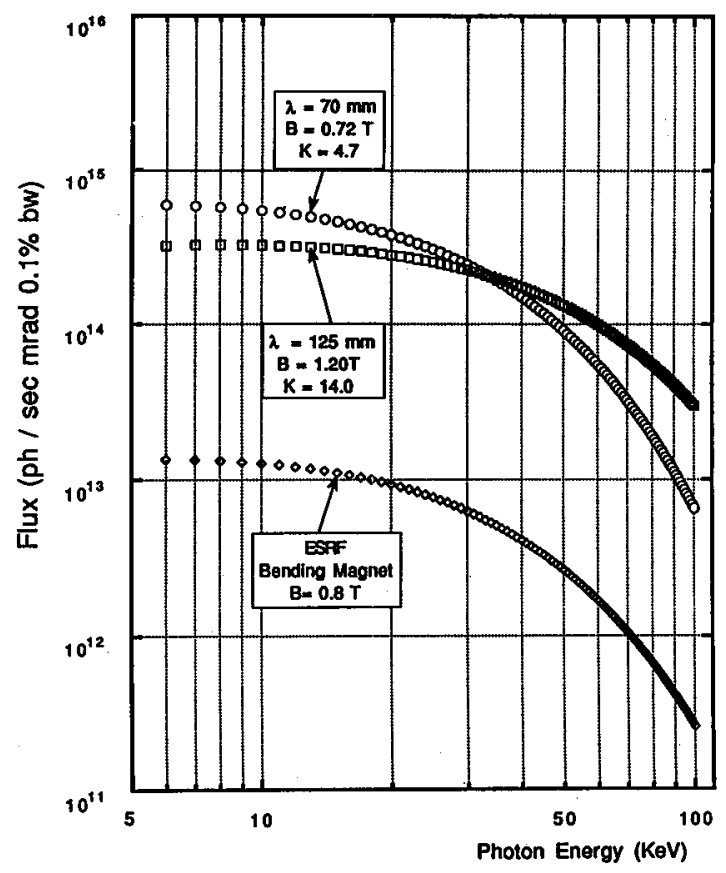

Fig. 2. The photon flux for wigglers 2 and 3 and from an ESRF bending magnet as function of photon energy.

\section{Beamline design}

In the design of experimental stations not only the brilliance, the photon energy, the flux, or the tunability of the radiation come into consideration. In designing beamlines using the new generation of insertion devices one of the most difficult problems is to control the heat load on the optical elements such as mirrors or monochromators. The insertion devices may generate radiating power in the multikilowatt regime. The total power integrated over phase space and photon energy by an insertion device can be calculated using the formula

$$
P[\mathrm{~kW}]=0.633 \cdot E^{2}[\mathrm{GeV}] \cdot B^{2}[\mathrm{~T}] \cdot L[\mathrm{~m}] \cdot I[\mathrm{~A}]
$$

where $L$ is the length of the insertion device and $I$ is the stored current. Note that the power changes as the square of the ring energy causing thermal heat load problems at the high-energy machines. At the ESRF power densities of up to $20-30 \mathrm{~W} / \mathrm{mm}^{2}$ at $30 \mathrm{~m}$ from the source will be found. In order not to degrade the brilliance of the source the optical elements have to be controlled typically within 5 microradians under the high heat loads.

The beamlines will thus, depending on the actual insertion device used, employ a variety of measures to control the heat load and the thermal expansion problems of the optical elements. Some of the more exotic measures include cryogenic cooling at liquid nitrogen temperatures of $\mathrm{Si}$ monochromator crystals and 
the use of adaptive feedback mirror technology using piezo-elements and autocollimators.

\section{Scientific program at the ESRF}

200 selected members of the European scientific community gathered in Grenoble in March 1989 to discuss the scientific priorities. The findings have been published as proceedings from the meeting [4]. The recommendation has resulted in a list of the 20 first beamlines to be constructed. See Table.

It can be seen from the list that the beamlines span a very wide range of applications from basic physics, molecular biology, chemistry to medicine.

The beamlines will be available free of charge with a selection procedure based on scientific merits. The experimental program is expected to start in the first half of 1994 .

\section{References}

[1] J.D. Jackson, Classical Electrodynamics, Wiley, New York 1962.

[2] ESRF Foundation Phase Report, European Synchrotron Radiation Facility, Grenoble 1987.

[3] P. Elleaume, Synchrotron Radiat. News 1, 18 (1988).

[4] Proc. ESRF User's Meeting, Grenoble 1989, European Synchrotron Radiation Facility, Grenoble 1989. 